\title{
Procesamiento del lenguaje natural con Python
}

\section{Natural language processing with Python}

CAMACHO-ÁLVAREZ, Miguel Ángel†* \& NAVARRO-ÁLVAREZ, Ernesto

Facultad de Ingeniería Electromecánica, Universidad de Colima

ID $1^{\mathrm{er}}$ Autor: Miguel Ángel, Camacho-Álvarez / ORC ID: 0000-0002-4823-2578

ID $1^{\mathrm{er}}$ Coautor: Ernesto, Navarro-Álvarez / ORC ID: 0000-0001-8236-3806

DOI: $10.35429 /$ JCA.2020.13.4.24.28

Recibido Enero 12, 2020; Aceptado Marzo 31, 2020

\section{Resumen}

En este trabajo, se presenta la tarea de desarrollar un chatbot para la página de Facebook de la Universidad de Colima que procese las peticiones de los usuarios en Lenguaje Natural (LN). Comúnmente, los chatbots solo procesan las peticiones preprogramadas. Para eliminar esta limitación, se propone desarrollar una Inteligencia Artificial (IA) que aprenda de las interacciones del usuario para poder procesar nuevas peticiones. Para realizar esto, se utilizó NLTK, la cuál es una librería de Python enfocada en el PLN que soporta el uso de IA. Todo esto, con la finalidad de reducir los tiempos de respuesta hacia los usuarios.

Procesamiento del Lenguaje Natural, Chatbot, Inteligencia Artificial

\begin{abstract}
In this work, we present the task of developing a chatbot that processes the user's requests in Natural Language (NL) that the Facebook Page of the University of Colima receives every day. Commonly, a chatbot only processes the preprogrammed requests. To remove this limitation, we propose to develop an Artificial Intelligence (AI) that learns through user interactions to process new requests. We use NLTK, which is a Python library that focuses on the NLP and support AI. For the purpose to reduce the response time to the users.
\end{abstract}

Natural Language Processing, Chatbot, Artificial Intelligence

Citación: CAMACHO-ÁLVAREZ, Miguel Ángel \& NAVARRO-ÁLVAREZ, Ernesto. Procesamiento del lenguaje natural con Python. Revista de Cómputo Aplicado. 2020. 4-13: 24-28.

\footnotetext{
* Correspondencia al Autor (Correo Electrónico: mcamacho3@ucol.mx)

$\dagger$ Investigador contribuyendo como primer autor.
} 


\section{Introducción}

En los últimos años, el Procesamiento del Lenguaje Natural (PLN) ha tenido un gran crecimiento gracias a la gran cantidad de datos que se pueden obtener a través de internet.

El PLN investiga el uso de computadoras para procesar o entender el lenguaje natural (LN) para el propósito de realizar tareas útiles. Se define como un campo interdisciplinario que combina la lingüística computacional, ciencias de la computación, ciencia cognitiva e Inteligencia Artificial (IA) [1].

La arquitectura de un sistema de PLN se divide en 5 niveles [2]:

- $\quad$ El fonológico se encarga de ver cómo las palabras se relacionan con los sonidos que representan.

- El morfológico revisa cómo las palabras se construyen de morfemas.

- El sintáctico que trata de cómo pueden unirse las palabras para formar oraciones.

- El semántico observa el significado de cada palabra y de cómo pueden unirse para dar significado a una oración.

- $\quad$ El pragmático donde se trata de cómo las oraciones se pueden utilizar en diferentes situaciones y cómo esto afecta en el significado de cada oración.

Una de las aplicaciones del PLN son los chatbots o agentes conversacionales los cuales se definen como sistemas computarizados que operan como una interfaz entre usuarios humanos y una aplicación de software, usando el $\mathrm{LN}$, ya sea hablado o escrito, como principal medio de comunicación [3]. Originalmente, el término chatbot se usó para referirse a programas de ordenador que intentaban simular el lenguaje humano con la ayuda de un sistema de diálogo basado en texto. Desde la introducción de los teléfonos inteligentes y las aplicaciones móviles, el término chatbot se utiliza principalmente para aplicaciones de mensajería en lugar de para programas informáticos puros.
Los chatbots son programas diseñados para interactuar con los usuarios de una manera similar a la humana, respondiendo preguntas y realizando tareas en un área específica [4].

Los chatbots han existido desde hace algunas décadas, pero su popularidad ha aumentado exponencialmente recientemente gracias a las mejoras en la IA, lo que permite que las respuestas de éstos sean más efectivas. Los ejemplos más conocidos de estos son Siri, IBM Watson, Alexa, etc. El problema básico que estos chatbots tratan de resolver es convertirse en un intermediario y ayudar a los usuarios hacerse más productivos [5].

Natural Language ToolKit (NLTK) es la plataforma líder para construir programas en Python con datos del lenguaje humano. Provee interfaces fáciles de usar con más de 50 cuerpos $\mathrm{y}$ recursos léxicos como WordNet, junto con un conjunto de librerías de procesamiento de texto para clasificación, tokenización, clasificación, parseo y razonamiento semántico [6].

NLTK fue diseñado teniendo en cuenta cuatro objetivos principales [7]:

\section{- $\quad$ Simplicidad.}

- Consistencia.

- $\quad$ Extensibilidad.

- $\quad$ Modularidad.

Una Red Neuronal (RN) es un modelo del campo de Machine Learning (ML). Este campo engloba todos los modelos computaciones que aprende de una experiencia $\mathbf{E}$ con respecto a alguna tarea $\mathbf{T}$, y alguna medida de rendimiento $\mathbf{P}$. Por tanto, una $\mathrm{RN}$ es un modelo de aprendizaje que representa un conjunto de operaciones interconectadas, simulando la sinapsis del cerebro humano, llamadas neuronas. Estas operaciones son compuestas por una entrada, una salida, y una operación no lineal. A su vez, sus formas de interconexión permiten aprender distintos patrones. Así como procesar el lenguaje, y sus peculiaridades, además de su secuencialidad implícita. [8] 


\section{Problema}

Los tiempos de respuesta de la página de Facebook de la Universidad de Colima suelen ser largos, ya que un operador humano no puede atender todas las solicitudes que recibe de parte de los usuarios. Además de los costos de operación que éste genera.

\section{Hipótesis}

La integración de un sistema de chatbot a la página de Facebook de la Universidad de Colima agilizará la consulta de información de parte de los usuarios y responderá correctamente las preguntas. El sistema se someterá a varias pruebas de confiabilidad como pruebas de estrés y pruebas reales con usuarios de prueba, para seguido de esto, implementarlo en la página.

\section{Objetivo General}

Desarrollar una IA para implementarla en un chatbot que procese los mensajes en LN que recibe a diario la página de Facebook de la Universidad de Colima, con la finalidad de que los usuarios puedan interactuar con él y hacer todo tipo de preguntas relacionas a la oferta educativa, programa de becas, calendario escolar, etc.

\section{Objetivos Específicos}

- Entender cómo funciona el PLN y poder aplicarlo en un chatbot.

- Aprender a desarrollar un chatbot utilizando las librerías NLTK de Python y TensorFlow de Google.

- Automatizar el proceso de consulta de información.

- Implementar el chatbot en la aplicación de mensajería Facebook Messenger para agilizar al tiempo de respuesta hacia los usuarios.

\section{Funcionamiento del sistema}

El funcionamiento del chatbot se muestra a continuación:

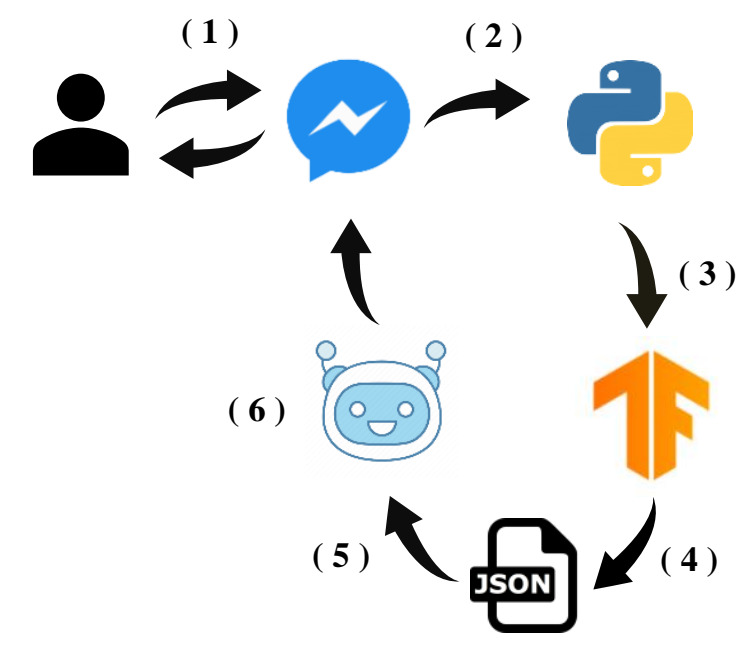

Figura 1 Diagrama del funcionamiento del sistema Fuente: elaboración propia

Como se muestra en la Figura 1, el proceso empieza con el usuario tecleando un mensaje en el servicio de mensajería de Facebook Messenger (1).

NLTK recibe el texto que escribió el usuario para tokenizarlo y crear una bolsa de palabras (2).

TensorFlow crea una red neuronal totalmente conectada y una activación softmax. La red neuronal analiza la bolsa de palabras que creó NLTK y compara que palabras existen en el vocabulario y cuáles no. La red se entrena 1000 veces (depende como se configure) y se guarda en un modelo para utilizarlo posteriormente (3).

El modelo busca en el archivo Json, que Intent tiene más aproximación y aleatoriamente se elige una respuesta (4).

La respuesta se manda al chatbot (5) y posteriormente, el chatbot manda la respuesta a Messenger (6) y se la manda al usuario (7).

\section{Resultados}

Se creó una página de Facebook para implementar el chatbot la cual se muestra a continuación: 


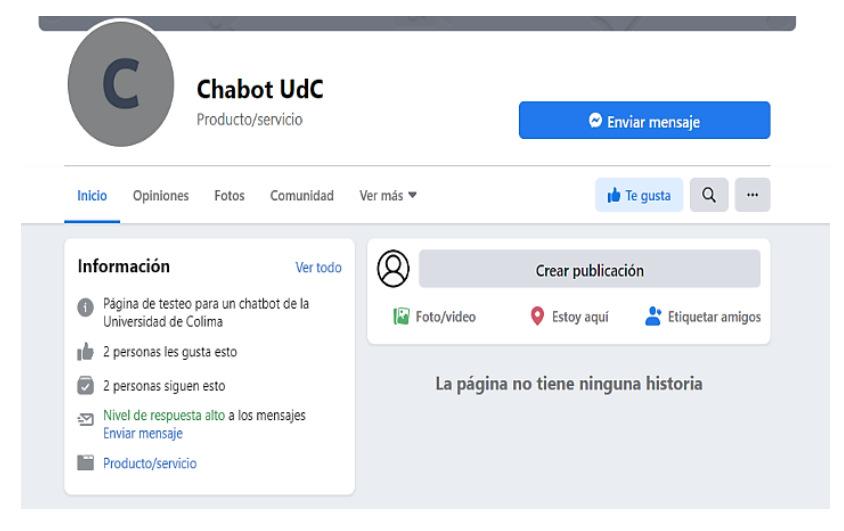

Figura 2 Página de Facebook donde se encuentra el chatbot

Al iniciar una nueva conversación, se muestra lo siguiente:
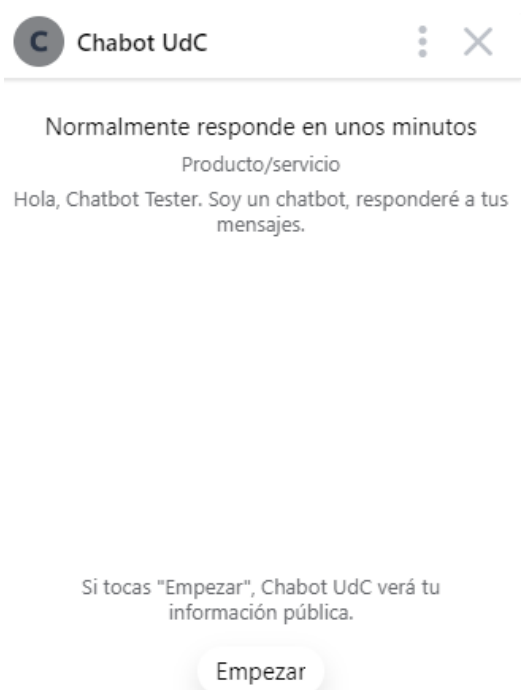

Figura 3 Pantalla de nueva conversación

Al clickear "Empezar" empieza a trabajar el chatbot:

C Chabot UdC : $\mathrm{X}$

Figura 4 Pantalla de Saludo del chatbot

Después del saludo, nos permite escribir lo que le vamos a preguntar:

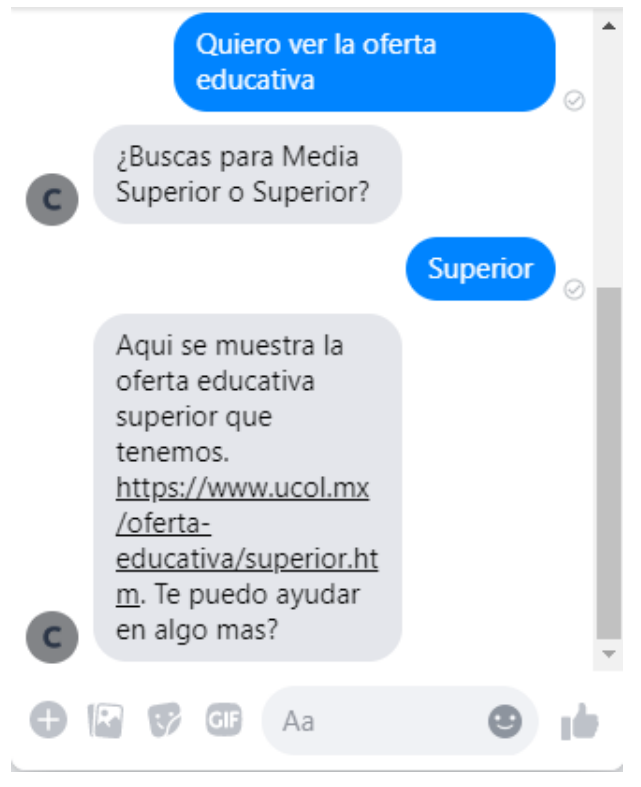

Figura 5 Pantalla de primera búsqueda del chatbot

Seguido de la primera respuesta, el chatbot, te pregunta si quieres realizar otra búsqueda, si la respuesta es positiva, puedes realizar otra consulta. En cambio, si la respuesta es negativa, se acaba la conversación.

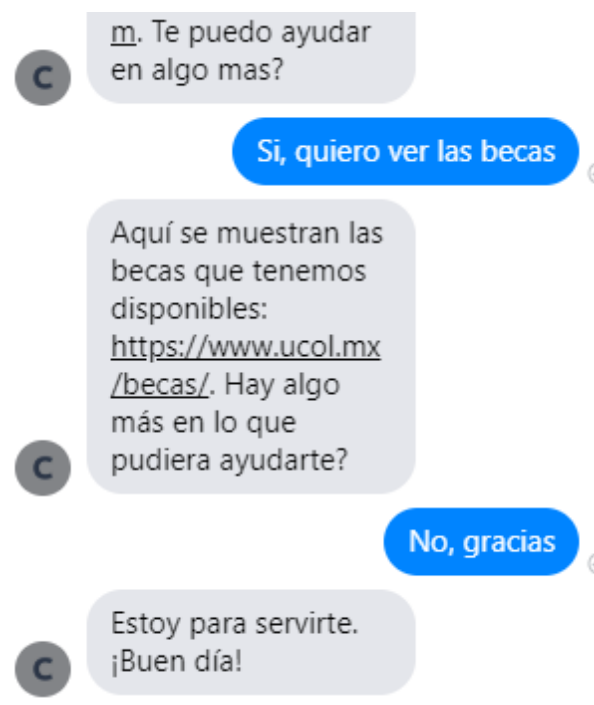

Figura 6 Pantalla de segunda búsqueda del chatbot.

\section{Conclusiones}

Gracias al desarrollo de este proyecto, se concluye que el uso de un chatbot dentro de una página de Facebook reduce el tiempo de respuesta, ya que, el usuario no tiene que esperar a un operador humano. 
Además, el uso de la IA mejora a la precisión del chatbot puesto que, al entrenar muchas veces los datos, las respuestas son más acertadas. Así mismo, complementa el chatbot, ya que al aprender nuevas interacciones amplía la cantidad de preguntas que puede responder.

\section{Referencias}

[1] Deng, L., \& Liu, Y. (Eds.). (2018). Deep learning in natural language processing. Springer.

[2] Vásquez, A. C., Quispe, J. P., \& Huayna, A. M. (2009). Procesamiento de lenguaje natural. Revista de investigación de Sistemas e Informática, 6(2), 45-54.

[3] Galitsky, B. (2019). Developing Enterprise Chatbots. Springer International Publishing.

[4] Gros Salvat, B., Escofet Roig, A., \& Payá Sánchez, M. (2020). Codiseño de un chatbot para facilitar procedimientos administrativos a población migrada. Pixel-Bit. Revista de Medios y Educación, 57, 91-106. https://doi.org/10.12795/pixelbit.2020.i57.03

[5] Raj, S. (2018). Building chatbots with Python. Using Natural Language Processing and Machine Learning. Apress.

[6] Natural Language Toolkit. Retrieved from https://www.nltk.org/

[7] Bird, S., Klein, E., \& Loper, E. (2009). Natural language processing with Python: analyzing text with the natural language toolkit. " O'Reilly Media, Inc.".

[8] Bragagnini Mendizábal, C. M. (2020). Traducción automática del español al inglés usando redes neuronales profundas con información conceptual de sentencias. 